Tecnología e Innovación

Tecnology and Innovation

Tecnologia e Inovação

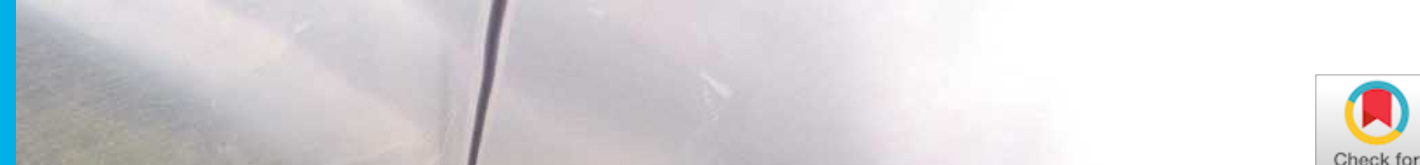

(n)
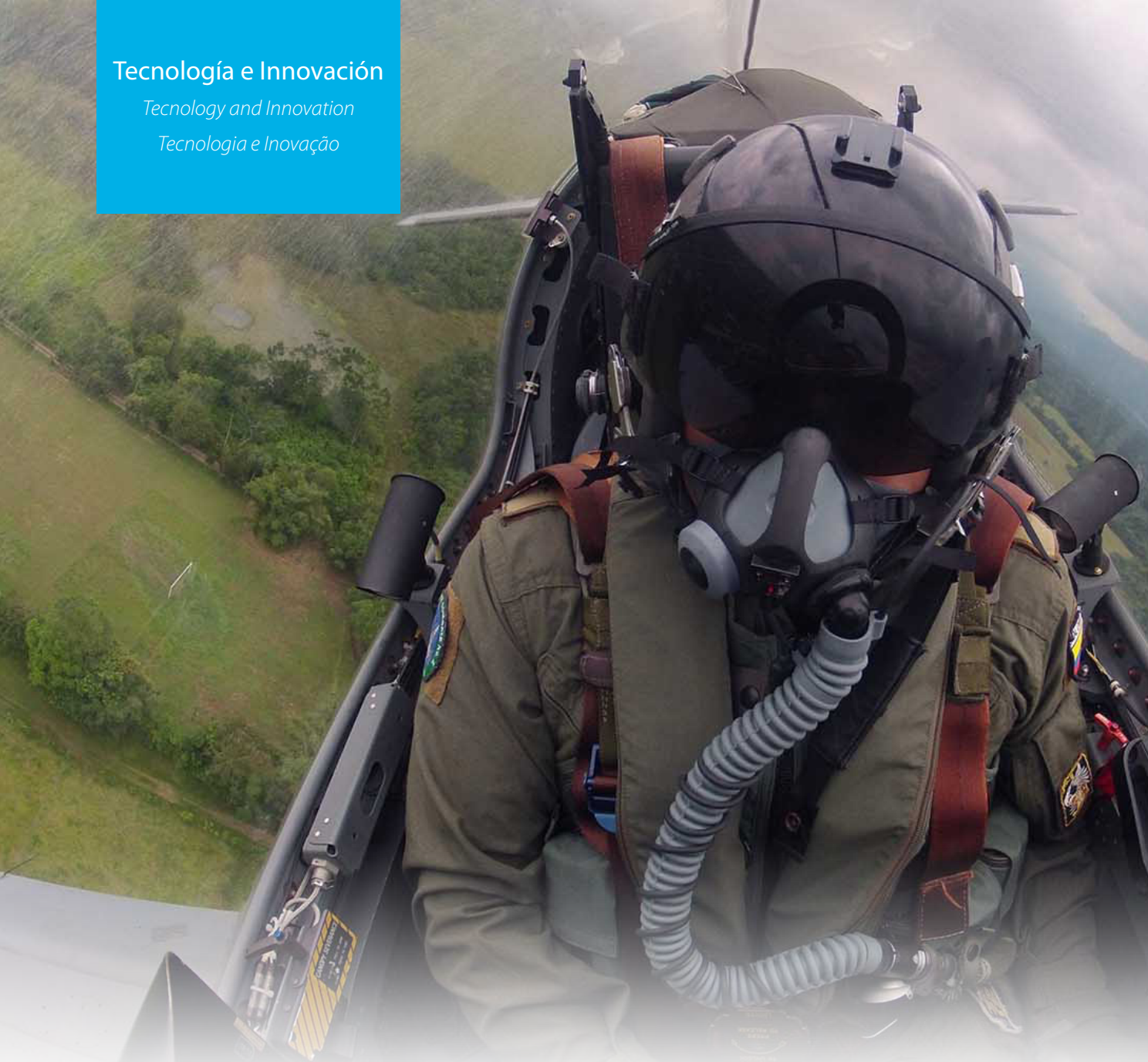

\title{
Aplicación del InTERnet de LAs Cosas en la SALUd: caso en la Enfermedad Pulmonar Obstructiva Crónica ${ }^{1}$
}

Internet of Things in healthcare: Case in Chronic Obstructive Pulmonary Disease ${ }^{2}$

A Aplicação da Internet das Coisas na saúde: no caso na Doença Pulmonar Obstrutiva Crônica 3

Leonardo Juan Ramírez López4, Andrés Fernando Marín López5 , Arturo Rodríguez Garcíaº

Universidad Militar Nueva Granada -UMNG. Bogotá, Colombia

Universidad Santiago de Chile. Santiago, Chile

\section{CIENCIA Y PODER AÉREO}

ISSN 1909-7050 / E- ISSN 2389-2468 / Volumen 13 / Número 1 / Enero-Junio de 2018 / Colombia / pp. 82-92 Recibido: 16/01/2017

Aprobado par evaluador: 06/02/2018

Doi: https://doi.org/10.18667/cienciaypoderaereo.589

82 | CIENCIA Y PODER AÉREO | Revista Científica de la Escuela de Postgrados de la Fuerza Aérea Colombiana 


\section{Para citar este artículo:}

Ramírez López, L. J., Marín López, A. F. y Rodríguez García, A. (2018). Aplicación del Internet de las Cosas en la salud: caso en la Enfermedad Pulmonar Obstructiva Crónica. Ciencia y Poder Aéreo, 13(1), 82-92. doi: https://doi.org/10.18667/cienciaypoder aereo.589

'Artículo científico original, que recoge los resultados de la implementación de tecnología loT para monitorizar variables fisiológicas de pacientes con enfermedades crónicas. Este proyecto pertenece a la línea de investigación de Telemetría del grupo de investigación en Telemedicina de la Universidad Militar Nueva Granada - TIGUM, financiado por la Universidad Militar Nueva Granada PIC-ING-2236.

${ }^{2}$ Original scientific article that collects the results of the internet of things technology to monitor physiological variables in patients with chronic diseases. This project belongs to the research lines of Telemetrics of the research group in telemedicine of Universidad Militar Nueva Granada, funded by Universidad Militar Nueva Granada PIC-ING-2236.

${ }^{3}$ Artigo científico original, que inclui os resultados da implementação da tecnologia loT para monitorar variáveis fisiológicas de pacientes com doenças crônicas. Este projeto pertence à linha de pesquisa em Telemetria do grupo de pesquisa em Telemedicina da Universidade Militar Nueva Granada - TIGUM, financiada pela Universidad Militar Nueva Granada. PICING-2236.

${ }^{4}$ Ingeniero electrónico, magíster en Ingeniería de Sistemas y doctor en Ingeniería Biomédica. Líder del grupo de investigación en Telemedicina de la Universidad Militar Nueva Granada - TIGUM, Bogotá, Colombia.Correo electrónico: tigum@unimilitar.edu.co

${ }^{5}$ Ingeniero en telecomunicaciones. Investigador del grupo TIGUM, de la Universidad Militar Nueva Granada, Bogotá, Colombia. Correo electrónico: tigum@unimilitar.edu.co

${ }^{6}$ Ingeniero mecánico-eléctrico, magíster en Telecomunicaciones, doctor en Ciencias de la Ingeniería. Líder del grupo de investigación en Nuevas Tecnologías - GINT-USACH, Santiago, Chile. Correo electrónico: arturo.rodriguez@usach.cl
Resumen: La Enfermedad Pulmonar Obstructiva Crónica - EPOC, corresponde a las diversas dolencias pulmonares crónicas que limitan el flujo de aire en los pulmones. Actualmente, presenta un importante aumento en su prevalencia y mortalidad a nivel mundial, siendo actualmente, según la Organización Mundial de la Salud, la cuarta causa de mortalidad en el mundo, donde más del $90 \%$ de estas muertes se producen en países de bajos y medianos ingresos. En Colombia es la cuarta causa de muerte, llegando en el 2016 a 38 muertes diarias. Por esto, el Plan Decenal de Salud Pública 2012-2021, establece las intervenciones que deben ser implementadas desde la prevención, detección temprana, tratamiento y rehabilitación. Esta investigación propone la implementación del Internet de las Cosas en una solución costo-efectiva para el monitoreo de esta enfermedad, mediante la medición constante de la temperatura corporal, saturación de oxígeno y frecuencia cardíaca con sensores de bajo costo que permitan analizar y emitir alarmas de prevención temprana. Los resultados permiten afirmar que todas las medidas realizadas con el Sistema loT desarrollado permanecen en el intervalo de confianza de los equipos tomados como referencia.

Palabras clave: EPOC, Internet de las Cosas, monitoreo, telemedicina.

Abstract: Chronic Obstructive Pulmonary Disease (COPD), which includes various chronic pulmonary diseases, causes limitations in lung airflow. COPD, now the fourth leading cause of death worldwide, poses a significant growth in its prevalence and mortality globally. According to the World Health Organization, more than $90 \%$ of COPD deaths occur in low or middle-income countries. In Colombia, it is the fourth cause of death totaling 38 deaths per day in 2012. Consequently, the ten-year Health Plan (2012-2022) establishes the interventions to be implemented from prevention, early detection, treatment and rehabilitation. This research aims to implement loT model within a cost-effective solution to monitor this disease through constant measurement of body temperature, oxygen saturation, and heart rate using low-cost sensors that permit to analyze and provide early prevention alarms. Results/Outcomes allow us to assert that all measurements conducted with this developed IoT System remain within confidence intervals in the equipment chosen/used as reference.

Keywords: COPD, Internet of Things, monitoring, telemedicine.

Resumo: A Doença Pulmonar Obstrutiva Crónica - DPOC, corresponde às várias doenças pulmonares crónicas, que limitam o fluxo de ar nos pulmões. Atualmente, apresenta um aumento significativo na sua prevalência e mortalidade a nível mundial, e é atualmente, de acordo com a Organização Mundial de Saúde, a quarta causa principal de morte no mundo, onde mais do $90 \%$ destas mortes ocorrem em países de baixa e média renda. Na Colômbia é a quarta causa de morte, em 2016 chegaram a acorrer 38 mortes por dia. Por isto mesmo, o Plano Decenal de Saúde Pública 2012-2021, estabelece as intervenções a serem implementadas a partir da prevenção, detecção precoce, tratamento e reabilitação. Esta pesquisa propõe a implementação da Internet das Coisas em uma solução de custo-eficaz para o monitoramento desta doença, através da medição constante da temperatura corporal, saturação de oxigênio e frequência cardíaca com sensores de baixo custo que permitam analisar e emitir alarmes de prevenção precoce Os resultados permitem conferir que todas as medidas tomadas com o sistema loT desenvolvido permanecem no intervalo de confiança dos equipamentos tomados como referência.

Palavras-chave: DPOC, Internet das Coisas, monitoramento, telemedicina. 


\section{Preliminares}

A continuación se explican algunos términos para la comprensión de la investigación desarrollada:

\section{Enfermedad Pulmonar Obstructiva Crónica - EPOC}

Según la Organización Mundial de la Salud - OMS, no es una sola enfermedad sino un concepto general que designa diversas dolencias pulmonares crónicas que limitan el flujo de aire en los pulmones, es prevenible, tratable y conduce a incapacidad e incluso la muerte (OMS, 2015a). Entre los factores de riesgo para desarrollar EPOC están el tabaquismo, la exposición a biomasa, la contaminación ambiental y la exposición laboral (contaminantes ambientales: ozono, partículas en suspensión (PM), monóxido de carbono (CO), dióxido de sulfuro (SO2), dióxido de nitrógeno (NO2) y otros gases), antecedente de tuberculosis, enfermedades respiratorias inferiores en la infancia y los factores genéticos (Ministerio de Salud, 2016).

\section{Internet de las Cosas - IoT}

Según la Organización Mundial de la Salud - OMS, no es una sola enfermedad sino un concepto general que designa diversas dolencias pulmonares crónicas que limitan el flujo de aire en los pulmones, es prevenible, tratable y conduce a incapacidad e incluso la muerte (OMS, 2015a). Entre los factores de riesgo para desarrollar EPOC están el tabaquismo, la exposición a biomasa, la contaminación ambiental y la exposición laboral (contaminantes ambientales: ozono, partículas en suspensión (PM), monóxido de carbono (CO), dióxido de sulfuro (SO2), dióxido de nitrógeno (NO2) y otros gases), antecedente de tuberculosis, enfermedades respiratorias inferiores en la infancia y los factores genéticos (Ministerio de Salud, 2016).

\section{Telemedicina}

Es la provisión de servicios de salud a distancia en los componentes de promoción, prevención, diagnóstico, tratamiento y rehabilitación, por profesionales de la salud que utilizan tecnologías de la información y la comunicación, que les permiten intercambiar datos con el propósito de facilitar el acceso y la oportunidad en la prestación de servicios a la población que presenta limitaciones de oferta, de acceso a los servicios o de ambos en su área geográfica (Ley 1419 de 2010).

\section{Introducción}

EI EPOC se caracteriza esencialmente por una limitación crónica al flujo aéreo poco reversible y asociada principalmente al humo de tabaco. Se trata de una enfermedad infradiagnosticada, con una elevada morbimortalidad y supone un problema de salud pública de gran magnitud (Miravitlles, Calle \& Soler-Cataluna, 2012). Se asocia a factores como el tabaquismo, la contaminación del aire e inhala- ción de productos químicos con una frecuencia constante (OMS, 2015a). Actualmente, estos factores han aumentado los índices de morbimortalidad y hacen que esta enfermedad se convierta en un problema de salud pública de gran magnitud (OMS, 2015b), que anualmente genera un gran gasto en salud (Chapman et al., 2006).

El estudio de prevalencia de la EPOC en Colombia realizado por la Fundación Neumológica Colombiana en el 2005, determinó que a nivel nacional 9 de cada 100 personas mayores de 40 años tenían EPOC, porcentaje que se distribuyó en 8.5 \% en Bogotá, 6.2 \% en Barranquilla, $7.9 \%$ en Bucaramanga, 8.5 \% en Cali y 13.5 \% en Medellín (Ministerio de Salud, 2016). Según cifras de mortalidad presentadas por el Departamento Administrativo Nacional de Estadística - DANE, en 2010, del total de muertes ocurridas en Colombia, cerca de 4.500 fueron por enfermedades crónicas de las vías respiratorias inferiores atribuidas al consumo de tabaco, incluido la EPOC (DANE, 2010).

La EPOC no es una enfermedad curable, pero es tratada para retrasar su progresión y mejorar la calidad de vida de las personas que la padecen. Habitualmente, el paciente es diagnosticado para iniciar una serie de pruebas de monitoreo de variables fisiológicas, las cuales causan alteraciones que pueden ocasionar la muerte. En el caso de monitorear la pulsoximetría, medida no invasiva de la saturación de oxígeno de la hemoglobina de la sangre arterial, se usa para prevenir la hipoxemia (Pauwels, Buist, Calverley, Jenkins \& Hurd, 2012).

Usando la telemedicina, como estrategia de cuidado y seguimiento a pacientes con EPOC, se requiere no solo de recursos económicos para dispositivos para su tratamiento y fármacos, sino además de recursos intangibles como el tiempo de entrenamiento al equipo de salud y a las familias que están al cuidado del paciente (De Aldana et al., 2014). Es por esto que la OMS promueve investigaciones que generen nuevas estrategias costo-efectivas, que involucren sistemas tecnológicos que fortalezcan la capacidad de los servicios de salud (OMS, 2012).

Con esta realidad, el grupo de investigación en Telemedicina de la Universidad Militar Nueva Granda, se ha propuesto mejorar el cubrimiento de las estrategias para el autocuidado de la salud, mediante el desarrollo de aplicaciones móviles (Ramírez y Parra, 2013) y diseñando dispositivos de bajo costo (Ramírez, Rodríguez y Cifuentes, 2014) para diferentes variables fisiológicas y de libre acceso a la sociedad. Llegando a proponer el servicio de telediagnóstico basado en la Arquitectura-Orientada a Servicios (Ramírez, Ubaque y Guillen, 2015). 


\section{Materiales y método \\ Materiales}

El sistema loT desarrollado para medición y seguimiento del EPOC, está compuesto de:

\section{Hardware}

Las características de los sensores utilizados se explican en la tabla 1. cos disponibles en tecnología Cloud Computing.

Tabla 1.

Características técnicas de los sensores utilizados en el sistema loT

\begin{tabular}{|c|c|c|c|}
\hline Características & Fabricante & De funcionamiento & Aspecto físico \\
\hline Temperatura & $\begin{array}{c}\text { Referencia: Exsense } \\
\text { Número de Modelo: MTS6k017C37C3935A } \\
\text { Tapa: de acero inoxidable } \\
\text { Recubrimiento: epoxi } \\
\text { Cable: separados de PTFE } \\
\text { Longitud del cable: } 9 F r-24 F r, 100-2000 \mathrm{~mm}\end{array}$ & $\begin{array}{c}\text { Salida: digital } \\
\text { Precisión: } \\
+/-0.1^{\circ} \mathrm{C} / 1 \sim 5 \%\end{array}$ & 7 \\
\hline Pulsoximetro & Referencia: CMS50DL & $\begin{array}{c}\text { Rango de medición SpO2: } 0 \text { \%-100 \% } \\
\text { Resolución: } 1 \% \text { para } \mathrm{SpO}_{2} \\
\text { Precisión: rango de error } \\
\text { del } 4 \% \text { para } \mathrm{SpO}_{2} \\
\text { Rango de medida FC: } \\
\text { 30bpm-250bpm, } \\
\text { Precisión: rango de error del } 2 \%\end{array}$ & 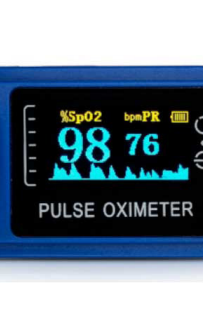 \\
\hline
\end{tabular}

Fuente: elaboración propia.

\section{Software}

Se desarrollaron programas para plataformas de "código abierto": Raspbian para Raspberry pi, Arduino 101, NetBeans IDE 8.1 y Ubuntu server 16.10 (Intel, 2016).

\section{Método}

Para esta investigación se utiliza el método exploratorio-descriptivo, que permite hacer un seguimiento al comportamiento del sistema propuesto. Se realizó en fases: en la primera fase se realiza una comparación de las soluciones existentes que incorporen tecnología loT en la telemedicina; con esta información se seleccionan los sensores, con base en: aplicación en salud, resolución de medida, portabilidad y costo. En la segunda fase se realiza el diseño y ensamble del sistema que incluya la conectividad a la web y a la tecnología Cloud Computing. Finalmente, en la tercera fase, se realiza el proceso de validación técnica y funcional del sistema desarrollado.

\section{Desarrollo \\ Referentes}

Se toma como referente el desarrollo de la "cuna inteligente que genera vínculos con la madre", que crea un ambiente que simula para los bebés prematuros la sensación de estar en el vientre de su madre, y permite a la madre escuchar y monitorear los signos vitales del bebé mediante métodos no invasivos (Agencia Informativa Conacyt, 2016). Otro referente fue el monitor de signos vitales, desarrollado para el hospital de Næstved, usado para monitorear pacientes con cáncer en su tratamiento de quimioterapia: frecuencia cardíaca, frecuencia respiratoria, temperatura corporal y postura, para prevenir niveles de infección por consecuencia de estos tratamientos (Consalud.es, 2016). Finalmente, se tomó el desarrollo de un loT personal que usa la interconexión de diferentes sensores para medir y hacer seguimiento de la obesidad desde la presión arterial y nivel de glucosa (Lee \& Ouyang, 2014). 
CIENCIA Y PODER AÉREO | ISSN 1909-7050 | E-ISSN 2389-9468 | Vol. 13 | Núm. 1| Ene - Jun 2018 | Escuela de Postgrados de la Fuerza Aérea Colombiana | pp 82-92

\section{Medición de las variables fisiológicas}

Saturación de oxígeno: el sensor permite ser instalado de forma no invasiva y muestra medición continua de oximetría de pulso. Con esta medida se hace el seguimiento periférico del porcentaje de hemoglobina saturada con oxígeno, por el paso de longitudes de onda específicas a través de la sangre - $\mathrm{SpO}_{2}$ (López-Herranz, 2003).
Frecuencia cardíaca: se mide la actividad eléctrica del corazón, mediante el registro gráfico del electrocardiograma y la frecuencia cardiaca.

Temperatura corporal: se mide el equilibrio entre la producción de calor por el cuerpo y su pérdida (Ramírez, Marín y Cifuentes, 2015), en el caso de EPOC se analiza esta variable para determinar la relación que tiene esta con la evolución de la enfermedad (Benítez, 2014).

En la tabla 2 presenta las características de los sensores de temperatura.

Tabla 2.

Características de los sensores de temperatura

\begin{tabular}{|c|c|c|c|}
\hline Sensor & Especificaciones & Precio (dólares) & Variable \\
\hline LM35 & $\begin{array}{l}\text { Rango - } 55 \text { a } 150^{\circ} \mathrm{C} \\
\text { Alta estabilidad } \\
\text { Voltaje } 4 \text { a } 30 \mathrm{~V} \\
\text { Precisión } \pm 0.5^{\circ} \mathrm{C}\end{array}$ & 2 USD & Análoga-no lineal \\
\hline Fluke infrarojo & $\begin{array}{c}\text { Rango }-30 \text { a } 400{ }^{\circ} \mathrm{C} \\
\text { Baja estabilidad } \\
\text { Voltaje } 9 \mathrm{~V} \\
\text { Precisión: tolerancia de hasta } \pm 1.5^{\circ} \mathrm{C}\end{array}$ & 30-40 USD & Digital \\
\hline $\begin{array}{l}\text { Termómetro } \\
\text { de mercurio }\end{array}$ & $\begin{array}{c}\text { Rango } 35 \text { a } 42{ }^{\circ} \mathrm{C} \\
\text { Alta estabilidad } \\
\text { No requiere baterías } \\
\text { Precisión: tolerancia de hasta } \pm 0.1^{\circ} \mathrm{C}\end{array}$ & 2-3 USD & Análoga \\
\hline Exsense & $\begin{array}{c}\text { Rango } 25 \text { a } 42{ }^{\circ} \mathrm{C} \\
\text { Alta estabilidad } \\
\text { Excelente resistencia térmica de ciclo } \\
\text { Precisión: tolerancia de hasta } \pm 0.5^{\circ} \mathrm{C}\end{array}$ & 10 USD & Análoga-lineal \\
\hline
\end{tabular}

Fuente: elaboración propia.

En la tabla 3, se presenta la comparación para los sensores de frecuencia cardiaca y saturación de oxígeno.

Tabla 3.

Comparación entre sensores de frecuencia cardiaca y saturación de oxígeno

\begin{tabular}{|c|c|c|c|}
\hline Sensor & Especificaciones & Precio (dólares) & Variable \\
\hline Datex OHMEDA & $\begin{array}{c}\text { Pantalla: no } \\
\text { Rango 0-100 } \\
\text { Voltaje 2.6-3.6 V } \\
\text { Precisión } \pm 1 \%\end{array}$ & 64 USD & Oxímetro \\
\hline Vitacare & $\begin{array}{c}\text { Pantalla: no } \\
\text { Rango 0-100 } \\
\text { Voltaje } 2.6-3.6 \mathrm{~V} \\
\text { Precisión } \pm 1 \%\end{array}$ & 119 USD & Oxímetro \\
\hline JZK-301 & $\begin{array}{c}\text { Pantalla: LED } \\
\text { Rango 0-100 } \\
\text { Voltaje 2.6-3.6 V } \\
\text { Precisión } \pm 2 \%\end{array}$ & 13 USD & Pulsoximetro \\
\hline
\end{tabular}

Fuente: elaboración propia. 


\section{Interconexión entre sensores}

Para realizar la interconexión de los sensores con las tarjetas Arduino-Raspberry Pi a la web y a Cloud para implementar la tecnología IoT, fue necesario adicionar una plataforma e-Health (E-Health Sensor, 2016), la cual es útil para realizar aplicaciones biométricas en telemedicina. La figura 1 muestra los sensores que pueden ser conectados a la plataforma e-Health.

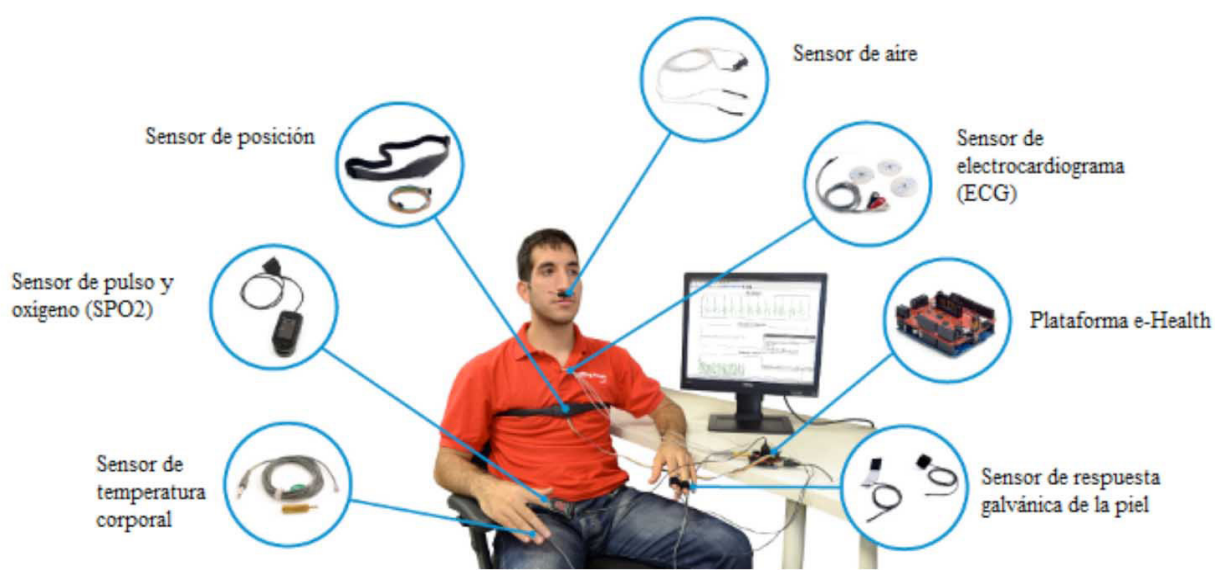

Figura 1. Plataforma e-Health Fuente: E-Health Sensor, 2016

La capacidad de sensores a instalar es: pulso cardiaco, oximetría (SPO2), flujo de aire (respiración), temperatura corporal, electrocardiograma (ECG), glucómetría, respuesta galvánica de la piel (GSR - sudoración), presión arterial (esfigmomanómetro) y posición del paciente (acelerómetro).

\section{Resultados}

Sistema de medición y seguimiento

El sistema diseñado se presenta en la figura 2. Se compone de cinco módulos que se explican a continuación:

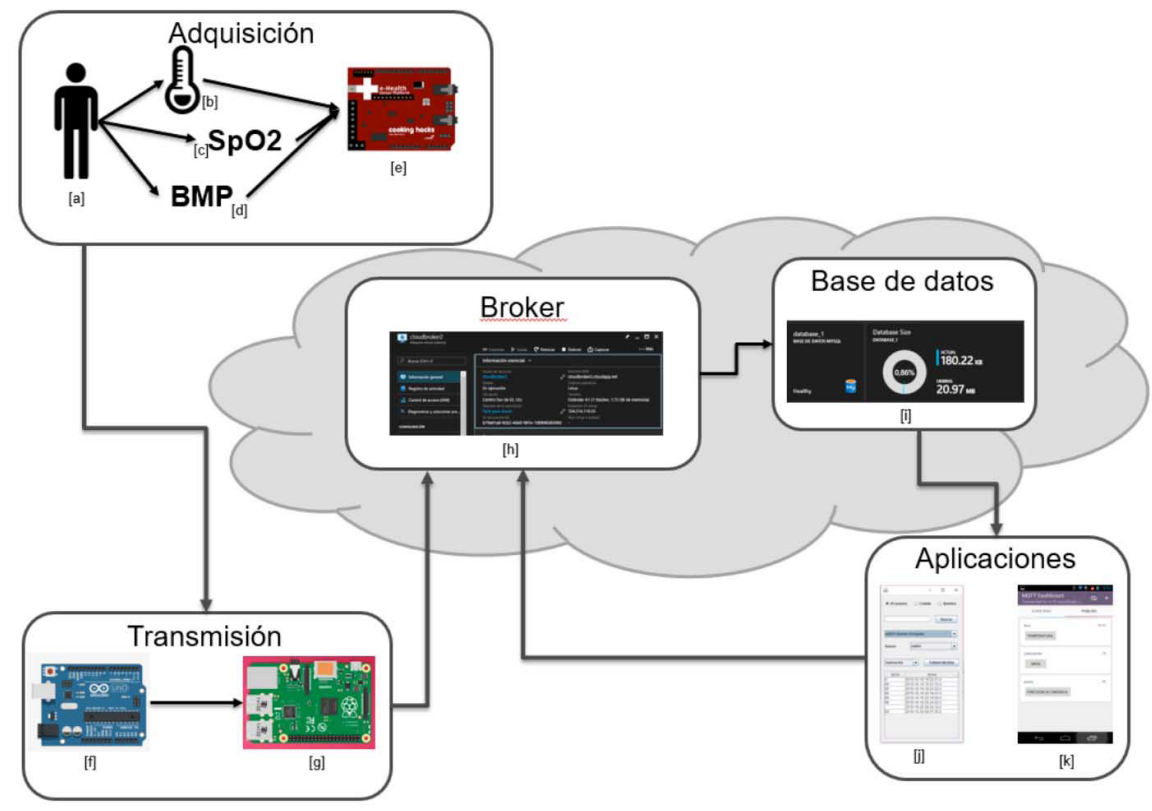

Figura 2. Sistema de medición y seguimiento diseñado Fuente: elaboración propia 
Módulo de adquisición de los datos: este módulo se compone de los siguientes elementos:

El paciente [a]: acorresponde a la persona diagnosticada con EPOC, en sus dos formas principales: la bronquitis crónica, la cual involucra una tos prolongada con moco; el enfisema, el cual involucra un daño a los pulmones con el tiempo.

El sensor de temperatura [b]: sensor de la plataforma e-Health que nos permite obtener los datos de temperatura corporal en un rango de $27-45^{\circ} \mathrm{C}$.

El sensor de saturación de oxigeno [c]: sensor de la plataforma e-Health que permite obtener los datos de saturación de oxígeno en un rango de 0-100\%.

El sensor de pulso cardíaco [d]: sensor de la plataforma e-Health que obtiene los datos del pulso cardiaco en un rango de 30-250bpm.

Plataforma e-Health [e]: conectada a las tarjetas Arduino y Raspberry Pi. A través de plataforma se obtienen los datos digitales de los sensores.

Módulo de transmisión: este módulo se compone de los siguientes elementos:

Arduino uno [f]: tarjeta y plataforma de código abierto. Se usó para comunicar de forma serial con la Raspberry Pi a una velocidad 9600 baudios sin paridad con un bit de parada y una trama de 8 bits.
Raspberry Pi [g]: tarjeta con microprocesador que permite la conexión entre el bróker en la nube y la plataforma Arduino. El agente bróker actúa como intermediario entre el comprador de un servicio de cloud computing y los vendedores de dicho servicio.

Módulo de comunicación: este módulo se compone de los siguientes elementos:

Comunicación con el bróker mqtt [h]: se realiza mediante el bróker Message Queue Telemetry Transport -mqtt [h]: usando el protocolo machine-to-machine -M2M, orientado a la comunicación entre sensores, debido a que consume muy poco ancho de banda y puede ser utilizado en la mayoría de los dispositivos. La comunicación se logra mediante suscripción y publicación de tópicos (Hunkeler, Truong \& Stanford-Clark, 2008).

Comunicación con la base de datos [i]: la base de datos fue creada para almacenar los datos adquiridos por los sensores, está suscrita a los tópicos usados en los sensores y los enviados por la Raspberry Pi.

La figura 3 muestra las conexiones entre los dispositivos y los clientes, utilizando el protocolo mqtt. En donde el mqtt cliente publica la información al bróker, que está configurado en la nube y suscrito para obtener los datos enviados y enviar los datos a los demás dispositivos suscritos al tópico.

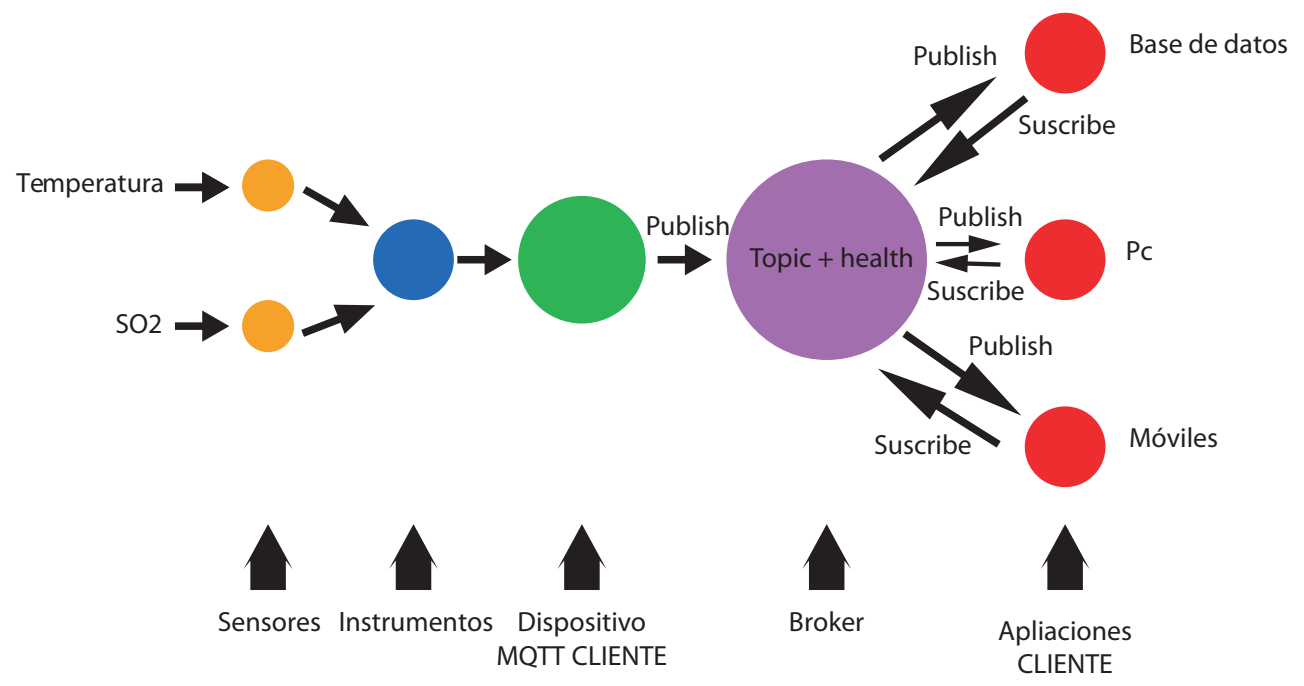

Figura 3. Conectividad entre dispositivos usando mqtt Fuente: elaboración propia 
Módulo de visualización: este módulo se compone de los siguientes elementos:

Aplicación en Java [j]: permite visualizar los datos en la base de datos mediante una interfaz gráfica, diseñada en NetBeans IDE 8.1.

Aplicación en Android [k]: se conecta al bróker, además permite agregar botones, modificar tópicos, publicaciones y suscriciones y generar una configuración personal para cada persona. Utiliza el protocolo mqtt, usuarios y contraseñas para garantizar la seguridad en la conexión. Las aplicaciones desarrolladas se muestran en la figura 4.
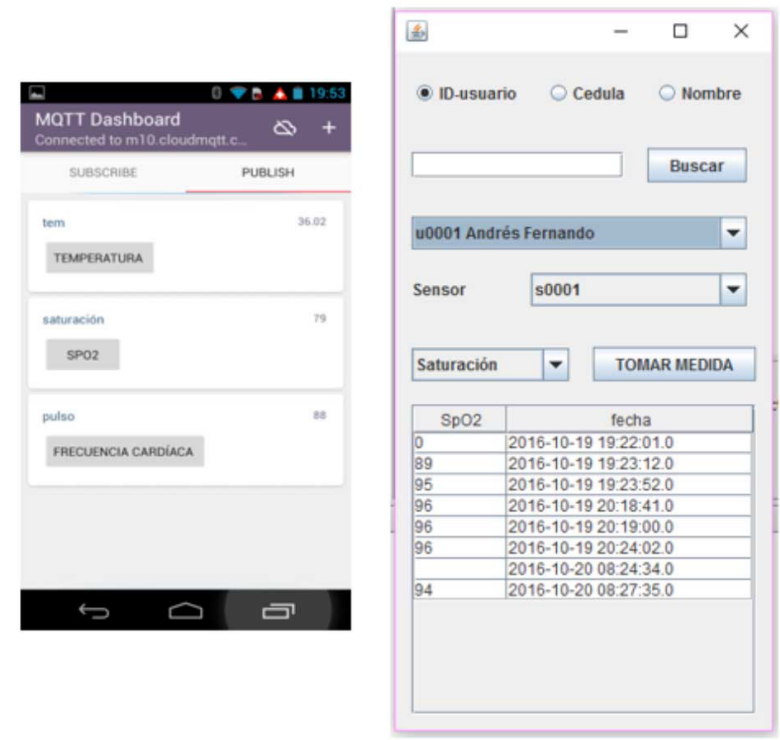

Figura 4. Aplicaciones loT que permiten la visualización de los datos

Fuente: elaboración propia
Módulo de conexión del sistema a internet: acordes con los avances de la tecnología, usamos las tarjetas Raspberry Pi (Raspberry-pi, 2016) por la valiosa cantidad de recursos para desarrollar diferentes soluciones tecnológicas, a través de un sistema con conexión a internet, proporcionado mediante la tecnología cloud computing, en donde son líderes empresas Amazon Web Services y Microsoft Azure. La figura 5 presenta la tendencia del mercado con relación al uso de tecnologías.

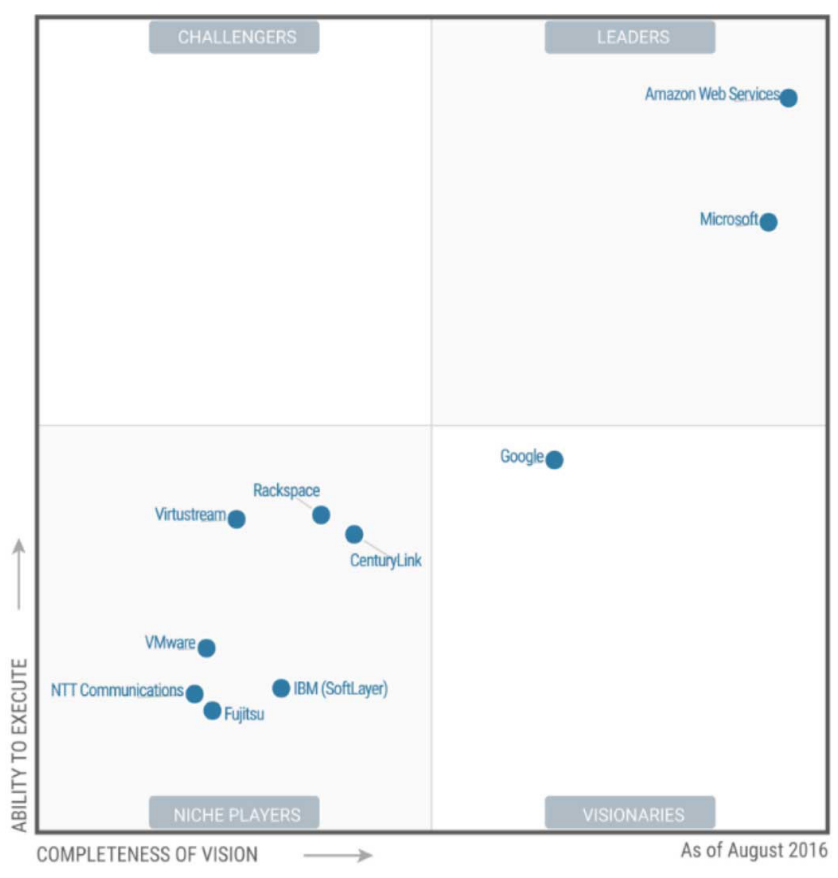

Figura 5. Cuadro mágico para servicios en la nube 2016 Fuente: Leong, Petri, Gill \& Dorosh, 2016 
CIENCIA Y PODER AÉREO | ISSN 1909-7050 | E-ISSN 2389-9468 | Vol. 13 | Núm. 1| Ene - Jun 2018 | Escuela de Postgrados de la Fuerza Aérea Colombiana | pp 82-92

\section{Validación}

Para verificar el adecuado funcionamiento del sistema IoT aplicado a la medición y seguimiento, se realizó un registro de datos de las variables de estudio y posteriormente se analizaron estadísticamente los datos, para verificar la precisión del sistema loT en comparación con otros dispositivos de medida patrón. La tabla 4 presenta los valores de las medidas realizadas con el sistema loT desarrollado y otros dispositivos.

Tabla 4.

Registro de datos adquiridos con el sistema loT y otros dispositivos

\begin{tabular}{|c|c|c|c|c|c|c|c|c|c|c|c|}
\hline \multicolumn{4}{|c|}{ Frecuencia cardíaca (pm) } & \multicolumn{4}{|c|}{ Saturación de oxigeno } & \multicolumn{4}{|c|}{ Temperatura } \\
\hline \multicolumn{2}{|c|}{ Sistema loT } & \multicolumn{2}{|c|}{ Agilent M3046A } & \multicolumn{2}{|c|}{ Sistema loT } & \multicolumn{2}{|c|}{ Agilent M3046A } & \multicolumn{2}{|c|}{ Sistema loT } & \multicolumn{2}{|c|}{ Agilent M3046A } \\
\hline 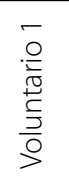 & 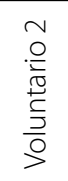 & 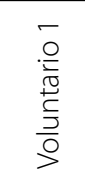 & 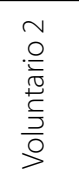 & $\begin{array}{l}\overline{-} \\
\stackrel{\circ}{\frac{0}{0}} \\
\stackrel{5}{=} \\
\stackrel{5}{\frac{J}{0}} \\
>\end{array}$ & 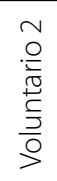 & 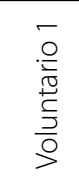 & 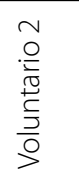 & 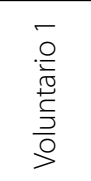 & 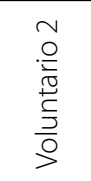 & 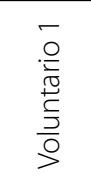 & $\begin{array}{l}N \\
\stackrel{0}{\circ} \\
\stackrel{0}{0} \\
\stackrel{0}{5} \\
\frac{3}{9} \\
>\end{array}$ \\
\hline 89 & 76 & 99 & 82 & 97 & 93 & 91 & 94 & 36.66 & 36.8 & 36.4 & 36.5 \\
\hline 86 & 76 & 101 & 75 & 97 & 93 & 92 & 94 & 36.21 & 35.4 & 36.4 & 35.2 \\
\hline 86 & 114 & 103 & 78 & 95 & 93 & 92 & 93 & 36.73 & 35.8 & 36.7 & 35.6 \\
\hline 86 & 114 & 101 & 80 & 93 & 93 & 92 & 93 & 36.9 & 36.23 & 36.8 & 35.9 \\
\hline 86 & 81 & 100 & 78 & 92 & 92 & 92 & 92 & 36.76 & 35.1 & 36.9 & 34.8 \\
\hline 87 & 81 & 100 & 79 & 93 & 92 & 92 & 91 & 35.25 & 35.55 & 35.5 & 35.3 \\
\hline 93 & 83 & 101 & 80 & 93 & 93 & 92 & 91 & 35.63 & 35.33 & 35.4 & 35.5 \\
\hline 96 & 85 & 100 & 80 & 94 & 94 & 92 & 91 & 34.6 & 35.31 & 34.2 & 35.2 \\
\hline 98 & 82 & 99 & 78 & 94 & 94 & 92 & 91 & 34.8 & 35.9 & 34.5 & 35.6 \\
\hline 98 & 80 & 102 & 79 & 94 & 94 & 92 & 92 & 34.5 & 36.33 & 35.4 & 35.5 \\
\hline 97 & 78 & 100 & 78 & 94 & 94 & 91 & 92 & 35.6 & 36.52 & 34.2 & 35.2 \\
\hline 100 & 79 & 101 & 80 & 93 & 94 & 91 & 92 & 34.4 & 35.26 & 34.5 & 35.6 \\
\hline 97 & 79 & 100 & 79 & 93 & 94 & 91 & 93 & 36.78 & 36.54 & 36.8 & 35.9 \\
\hline 93 & 79 & 101 & 78 & 93 & 94 & 91 & 92 & 36.15 & 35.8 & 36.8 & 35.9 \\
\hline 98 & 80 & 102 & 79 & 93 & 94 & 91 & 92 & 36.15 & 35.85 & 36.9 & 34.8 \\
\hline
\end{tabular}

Fuente: elaboración propia. Programa MATLAB R2009b

Ahora se realiza el análisis estadístico: desviación estándar y el error porcentual. La tabla 5 presenta los valores de la desviación de la medida.

Tabla 5 .

Análisis de datos adquiridos con el sistema loT y otros dispositivos de referencia: valores porcentuales

\begin{tabular}{|c|c|c|c|c|c|c|}
\hline \multirow{2}{*}{$\frac{\text { Variable }}{\text { Equipos referencia }}$} & \multicolumn{2}{|c|}{ Frecuencia cardíaca } & \multicolumn{2}{|c|}{ Saturación de oxigeno } & \multicolumn{2}{|c|}{ Temperatura } \\
\hline & Voluntario & Voluntario & Voluntario & Voluntario & Voluntario & Voluntario \\
\hline Agilent M3046A & 96.27 & 98.97 & 99.66 & 99.32 & & \\
\hline Begut MT 402 & & & & & 99.31 & 98.67 \\
\hline Sistema loT & 93.37 & 92.02 & 99.02 & 99.5 & 99.39 & 99.65 \\
\hline
\end{tabular}

Fuente:elaboración propia. Programa MATLAB R2009b 
La Tabla 6 presenta la estimación del intervalo de confianza en la medida de los equipos de referencia y del sistema loT desarrollado.

Tabla 6.

Estimación de la confianza en la medida de los equipos y del sistema loT

\begin{tabular}{ccccccc}
\hline \multicolumn{1}{c}{ Variable } & \multicolumn{2}{c}{ Frecuencia cardíaca } & \multicolumn{2}{c}{ Saturación de oxigeno } & \multicolumn{2}{c}{ Temperatura } \\
\hline Confianza en la medida & Voluntario & Voluntario & Voluntario & Voluntario & Voluntario & Voluntario \\
\hline $\begin{array}{c}\text { Intervalo para equipos } \\
\text { referencia }\end{array}$ & $99.27-96.36$ & $98.97-92.02$ & $99.66-99.02$ & $99.50-99.32$ & $99.39-99.31$ & $99.67-99.50$ \\
\hline Sistema loT desarrollado & 97.09 & 93.05 & 99.36 & 99.82 & 99.92 & 99.02 \\
\hline
\end{tabular}

Fuente: elaboración propia. Programa MATLAB R2009b

Los intervalos de confianza en las medidas de los equipos tomados como referencia para las variables seleccionadas se analizan desde las desviaciones de las 15 repeticiones en mediciones realizadas a cada voluntario. El intervalo de confianza en la medida de los equipos tomados como referencia, permite asegurar que todas las medidas realizadas con el sistema loT desarrollado permanecen en el intervalo de confianza de los equipos tomados como referencia.

\section{Conclusiones}

- Se ofrece una solución tecnológica para el seguimiento de pacientes diagnosticados con EPOC, que usa la tecnología loT para la interconexión de los sensores, el almacenamiento de las mediciones en Cloud Computing y su visualización a través de un aplicativo móvil o acceso web.

- Al realizar un estudio de benchmarking para comprar el sistema loT ofrecido con otras soluciones disponibles, logramos el objetivo de ser la solución de más bajo costo (al 50 \% de la más económica) y así lograr reducir el costo de la atención médica domiciliaria y aumentando la accesibilidad de la información médica.

- Con base en el criterio intervalo de confianza, usado para comparar las medidas dl nuevo sistema con el patrón, los resultados demuestran que el uso del internet de las cosas en aplicaciones de salud es útil y continua en desarrollo para nuevas aplicaciones.

- Una ventaja importante del sistema loT presentado, es la gestión eficiente de un gran volumen de datos utilizando Cloud Computing y la próxima aplicación de técnicas de inteligencia artificial para caracterizar y crear estándares predictivos, que permitan al sistema reportar en tiempo real a los profesionales de la salud cambios inesperados en signos vitales o condiciones que puedan ubicar en riesgo la salud de los pacientes.
Esta investigación abre las puertas a nuevas soluciones para el monitoreo en atención domiciliaria; i) uso de otros sensores y otros tipos de mediciones; ii) seguimiento a otras patologías que atenten contra la salud pública de nuestra sociedad; iii) uso del protocolo de comunicación mqtt utilizado en el proceso de conexión al sistema loT para la seguridad de la información; y iv) nuevas redes de comunicación entre los sensores y su interacción con el medio ambiente del paciente.

\section{Reconocimientos}

El desarrollo de este trabajo de investigación fue posible gracias al apoyo financiero proporcionado por la Universidad Militar Nueva Granada, mediante el proyecto de investigación PIC-ING-2236 "IoT aplicado en la Telemedicina" y el profesor doctor Arturo Rodríguez García de la USACH, en la validación del sistema loT.

\section{Referencias}

Agencia Informativa Conacyt. (2016). Crean una cuna inteligente que genera vínculos con la madre. Recuperado de http://www.conacytprensa.mx/index.php/tecnologia/tic/2333-desarrollancuna-inteligente-que-recrea-vinculos-afectivos-madre-hijo

Benítez, C. (2014). Enfermedades por calor e hidratación deportiva. Recuperado de https://docs.wixstatic.com/ugd/90d83e_ca5fd3b59bed4ca0b2096351579b8c06.pdf

Chapman, K., Mannino, D., Soriano, J., Vermeire, P., Buist, A., Thun, M., \& Aldington, S. (2006). Epidemiology and costs of chronic obstructive pulmonary disease. European Respiratory Journal, 27(1), 188-207.

Consalud.es. (2016). Un sensor 'todoterreno' puede mejorar la eficacia de los tratamientos contra el cáncer. Recuperado de http:// consalud.es/tecnologia/un-sensor-todoterreno-puede-mejorar-la-eficacia-de-los-tratamientos-contra-el-cancer-25498 
CIENCIA Y PODER AÉREO | ISSN 1909-7050 | E-ISSN 2389-9468 |Vol. 13 | Núm. 1| Ene - Jun 2018 | Escuela de Postgrados de la Fuerza Aérea Colombiana | pp 82-92

De Aldana, M., Plata, D., Matajira, J., y Niño, E. (2014). Las enfermedades crónicas no transmisibles y el uso de tecnologías de información y comunicación: revisión sistemática. Revista Cuidarte, 5(1), 661-669.

Departamento Administrativo Nacional de Estadística - DANE. (2010). Investigación de causas de las enfermedades no transmisibles. Recuperado de https://www.dane.gov.co/files/investigaciones/poblacion/defunciones/causa_defuncion/causas_ defuncion_2010.xls

E-Health Sensor Platform V2.0 for Arduino and Raspberry Pi. (2016). Biometric / Medical Applications. Recuperado de: https:// www.cooking-hacks.com/documentation/tutorials/ehealthbiometric-sensor-platform-arduino-raspberry-pi-medical

Hunkeler, U., Truong, H., \& Stanford-Clark, A. (2008). MQTT-SA publish/subscribe protocol for Wireless Sensor Networks. Communication systems software and middleware and workshops, pp. 791-798. IEEE.

Intel ${ }^{\circledR}$ Galileo Board. (2016). Discontinued Maker \& Innovator Products. Retrieved from https://software.intel.com/en-us/iot/ hardware/galileo

Lee, B., \& Ouyang, J. (2014). Intelligent Healthcare Service by using Collaborations between IoT Personal Health Devices. Blood pressure, (10), 11.

Leong, L., Petri, G., Gill, B., \& Dorosh, M. (2016). Magic Quadrant for Cloud Infrastructure as a Service, Worldwide. Retrieved from https://www.gartner.com/doc/reprints?id=1-2G2O5FC\&ct $=150519$

Ley 1419. (13 de diciembre de 2010). Por la cual se establecen los lineamientos para el desarrollo de la Telesalud en Colombia. Diario Oficial n. 0 47.922. Recuperado de https://docs.supersalud.gov. co/PortalWeb/Juridica/Leyes/L1419010.pdf
López-Herranz, G. P. (2003). Oximetría de pulso: a la vanguardia en la monitorización no invasiva de la oxigenación. Revista Médica del Hospital General de México, 66(3), 160-169.

Ministerio de Salud y Protección Social de Colombia. (2016). Enfermedad pulmonar obstructiva crónica (EPOC). Recuperado de https://www.minsalud.gov.co/salud/publica/PENT/Paginas/ Enfermedad-pulmonar-obstructiva-cronica.aspx

Miravitlles, M., Calle, M., \& Soler-Cataluna J. (2012). Clinical phenotypes of COPD: Identification, definition and implications for guidelines. Arch Bronconeumol, 48(3), 86-98.

Organización Mundial de la Salud - OMS. (2012). Estrategia para la prevención y el control de las enfermedades no transmisibles. recuperado de http://apps.who.int/gb/archive/pdf_files/ WHA53/sa14.pdf

Organización Mundial de la Salud - OMS. (2015a). ¿Qué es la enfermedad pulmonar obstructiva crónica (EPOC)? Recuperado de http://www.who.int/features/qa/48/es/

Organización Mundial de la Salud - OMS. (2015b). Enfermedad pulmonar obstructiva crónica (EPOC). Recuperado de http://www. who.int/mediacentre/factsheets/fs315/es/

Pauwels, R., Buist, A., Calverley, P., Jenkins, C., \& Hurd, S. (2012). Global strategy for the diagnosis, management, and prevention of chronic obstructive pulmonary disease. American journal of respiratory and critical care medicine.

Ramírez, L., y Parra, D. (2013). SMCa: Sistema de Monitoreo Móvil Cardiaco. Ciencia y Poder Aéreo, 8(1), 91-96. doi: https://doi. org/10.18667/cienciaypoderaereo.10

Ramírez, L., Rodríguez, Y., y Cifuentes, Y. (2014). Prototipo de electrocardiógrafo bipolar para uso académico. Ciencia y Poder Aéreo, 9(1), 115-123. doi: https://doi.org/10.18667/cienciaypoder aereo. 140 


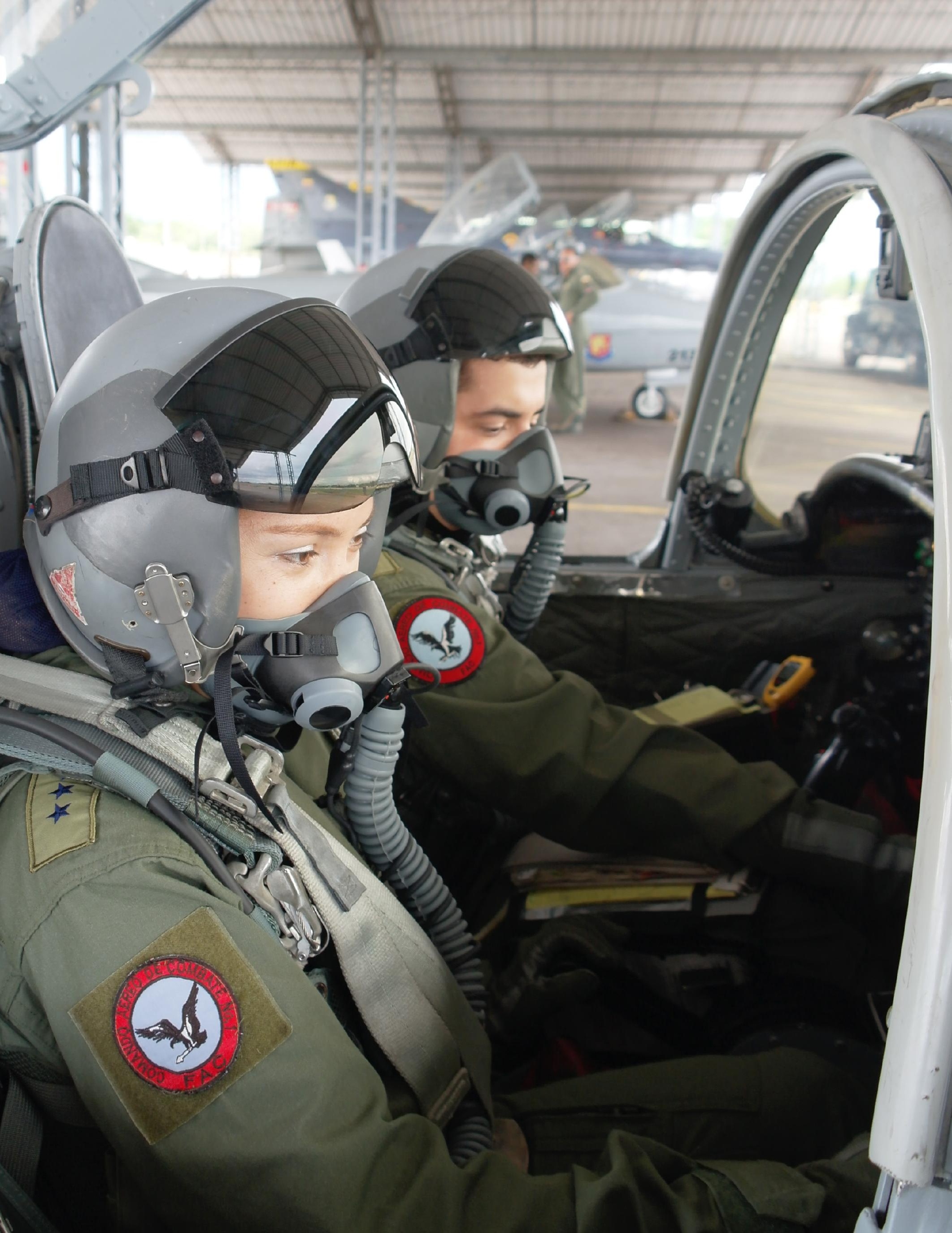

